\title{
Long-term cardiovascular outcomes in type 1 diabetic patients after simultaneous pancreas and kidney transplantation compared with living donor kidney transplantation
}

\author{
Jørn P. Lindahl ${ }^{1,2}$ • Anders Hartmann ${ }^{1,2} \cdot$ Svend Aakhus $^{3} \cdot$ Knut Endresen $^{3}$. \\ Karsten Midtvedt $^{2} \cdot$ Hallvard Holdaas $^{2} \cdot$ Torbjørn Leivestad $^{2} \cdot$ Rune Horneland $^{2}$ • \\ Ole Øyen ${ }^{2} \cdot$ Trond Jenssen ${ }^{2,4}$
}

Received: 28 October 2015 / Accepted: 14 December 2015 /Published online: 28 December 2015

(C) Springer-Verlag Berlin Heidelberg 2015

\begin{abstract}
Aims/hypothesis Mortality due to cardiovascular disease (CVD), particularly coronary artery disease (CAD), is high in type 1 diabetic patients with end-stage renal disease (ESRD). We aimed to determine whether normoglycaemia, as achieved by successful simultaneous pancreas and kidney (SPK) transplantation, could improve long-term outcomes compared with living donor kidney-alone (LDK) transplantation.

Methods We studied 486 type 1 diabetic patients with ESRD who underwent a first SPK $(n=256)$ or LDK $(n=230)$ transplant between 1983 and 2012 and were followed to the end of 2014. Data were retrieved from the Norwegian Renal Registry and hospital records. Kaplan-Meier plots and multivariate Cox regression, with correction for recipient, donor and transplant factors, were used to examine potential associations between transplant type and all-cause and CVD- and CADrelated mortality.

Results Median follow-up time was 7.9 years (interquartile range 4.3, 12.9). The adjusted HR for CVD-related deaths in SPK recipients compared with LDK recipients was 0.63 (95\% CI $0.40,0.99 ; p=0.047)$, while the HRs for all-cause and
\end{abstract}

Jørn P. Lindahl

j.p.h.lindahl@medisin.uio.no

Institute of Clinical Medicine, University of Oslo, Oslo, Norway

2 Department of Transplant Medicine, Oslo University Hospital, Rikshospitalet, Sognsvannsveien 20, 0372 Oslo, Norway

3 Department of Cardiology, Oslo University Hospital, Rikshospitalet, Oslo, Norway

4 Metabolic and Renal Research Group, UiT The Arctic University of Norway, Tromsø, Norway
CAD-related mortality were 0.81 (95\% CI $0.57,1.16$; $p=0.25)$ and $0.63(95 \% \mathrm{CI} 0.36,1.12 ; p=0.12)$, respectively. Compared with the LDK group, SPK recipients were younger and received grafts from younger donors. Cardiovascular mortality was higher in patients transplanted between 1983 and 1999 compared with those who received their grafts in subsequent years.

Conclusions/interpretation In patients with type 1 diabetes and ESRD, SPK transplantation was associated with reduced long-term cardiovascular mortality compared with LDK transplantation.

Keywords Cardiovascular disease · Diabetic nephropathy · Kidney transplantation $\cdot$ Pancreas transplantation · Type 1 diabetes
Abbreviations
CAD Coronary artery disease
CBVD Cerebrovascular disease
CVD Cardiovascular disease
ESRD End-stage renal disease
IQR Interquartile range
KTA Kidney transplantation alone
LDK Living donor kidney
PAD Peripheral artery disease
SPK Simultaneous pancreas and kidney

\section{Introduction}

Patients with end-stage renal disease (ESRD) suffer higher rates of cardiovascular morbidity and mortality than the general population $[1,2]$. Diabetes mellitus remains one of the 
most significant risk factors for development of ESRD [3]. For patients with ESRD, kidney transplantation is in general the treatment of choice, offering improved survival compared with continuous dialysis [4]. Cardiovascular complications and coronary artery disease (CAD) remain the predominant causes of death following successful kidney transplantation [5-7]. In patients with both type 1 diabetes and ESRD, simultaneous pancreas and kidney (SPK) transplantation has become an established treatment [8-10]. The obvious benefit of a functioning pancreas graft is normalised blood glucose control without the use of insulin, compared with consistent moderate hyperglycaemia controlled with insulin use. The question whether cardiovascular outcomes can be improved by transplanting a pancreas simultaneously with the kidney remains, however, controversial [11-14]. A major obstacle when comparing outcomes following SPK vs kidney transplantation alone (KTA; from either a deceased or a living donor) in recipients with type 1 diabetes has been imbalance in risk factors between patient populations, particularly due to selection of younger patients with less comorbidity for SPK transplantation. At our centre we have promoted the use of living donor kidney (LDK) transplantation for patients with type 1 diabetes [15]. Today, $30-40 \%$ of all our kidney transplantations are from living donors; patients tend to be younger and with lower comorbidity than those selected for transplantation with a kidney from a deceased donor. Thus, they serve as a suitable comparator to recipients receiving an SPK transplant.

We investigated recipients with known type 1 diabetes and addressed the question whether long-term normoglycaemia, as achieved by successful SPK transplantation, could reduce post-transplant cardiovascular mortality when compared with LDK transplantation alone.

\section{Methods}

Patients In Norway all organ transplantations are performed at a single centre: Oslo University Hospital, Oslo, Norway. Annually we perform between 250-300 kidney and 30-40 pancreas transplantations. We analysed data from the Norwegian Renal Registry and studied medical records as an additional source to obtain more complete comorbidity data. All patients $>18$ years of age with type 1 diabetes $(n=486)$ who had a first transplantation with a combined graft (SPK, $n=256$ ) or a kidney graft from a living donor (LDK, $n=230$ ) from January 1983 through December 2012 were eligible. Recipients were followed for clinical outcomes to the end of 2014 , i.e. minimum 2 years post engraftment. Causes of death were defined according to the European Renal AssociationEuropean Dialysis and Transplant Association (ERA-EDTA) coding systems [16]. No patient was lost to follow-up. The study was approved by the regional committee for medical and health research ethics.

Cardiac assessment prior to transplantation Since 1999, coronary angiography has been included as a routine part of the cardiac work-up of all patients with type 1 diabetes who were potential transplant recipients [17]. Before 1999, patients were screened for ischaemic heart disease with a non-invasive cardiac stress test, and, if judged clinically relevant, coronary angiography was performed. According to our transplantation protocol, we do not accept patients with an ejection fraction of less than $30 \%$.

Evaluation of cardiovascular comorbidity prior to transplantation Cardiovascular comorbidity among SPK and LDK recipients was classified as $\mathrm{CAD}$, cerebrovascular disease (CBVD) and/or peripheral artery disease (PAD). CAD was defined as coronary artery luminal diameter stenosis at or above $50 \%$ in at least one segment, using a 16-segment American Heart Association coronary artery classification, with or without revascularisation, including percutaneous coronary intervention and/or coronary artery bypass grafting, and/or having a myocardial infarction [18]. CBVD was defined as having symptoms and/or radiographic findings consistent with stroke. PAD was defined as intermittent claudication and/or need for percutaneous transluminal angioplasty in the lower extremity and/or amputation.

Surgical techniques At our centre, pancreas transplantation has been performed since 21 June 1983; initially, a ductoccluded segmental pancreas was used for transplantation [19]. From April 1988, this technique was replaced by an unoccluded pancreas graft where the exocrine secretion was drained by an anastomosis of a duodenal segment from the donor to the urinary bladder [19]. After March 1998, bladder drainage was substituted with enteric drainage through a donor-derived duodenal segment attached to the proximal jejunum [20]. Since September 2012, the duodenal part of the transplant has been anastomosed to the recipient's duodenum rather than the jejunum to obtain endoscopic biopsies from both the duodenum and pancreas allografts [21].

Since December 2004, an increasing number of kidney transplant recipients have been subjected to minimally invasive kidney transplantation compared with previous conventional kidney transplantation [22].

Immunosuppressive drugs The immunosuppression protocols have changed over time. In the period 19831999, kidney transplant recipients mainly received maintenance immunosuppression with prednisolone, ciclosporin and azathioprine. Recipients transplanted after year 2000 have in addition to maintenance immunosuppression received induction therapy with 
antithymocyte antiglobulin (SPK) or basiliximab (KTA). Azathioprine has been replaced by mycophenolate mofetil since the late 1990s, and patients have primarily received tacrolimus at the expense of ciclosporin as a calcineurin inhibitor. Target levels for immunosuppressive drugs have been higher for recipients of an additional pancreas transplant compared with those receiving a single kidney.

To look at change over time, and since some changes in the transplant protocol coincided roughly with the year 2000, we divided the study population into an early era (1983-1999) and a recent era (2000-2012).

Statistical analysis Demographic data were summarised and grouped by mode of treatment. Continuous variables are reported as the mean $\pm \mathrm{SD}$ or median (interquartile range [IQR]) depending on normality fit. Categorical data are described using frequencies. Student's $t$ test for independent samples or the Mann-Whitney $U$ (Wilcoxon) test was used to compare continuous variables, as appropriate. Categorical variables were compared using Pearson $\chi^{2}$ test (Fisher's exact test was applied if the number of observations per cell was fewer than five). Kaplan-Meier curves were used to construct probability of no all-cause and cardiovascular disease (CVD)and CAD-related deaths. Cox proportional hazards models were used to calculate unadjusted and adjusted HRs for allcause and CVD- and CAD-related deaths in relation to treatment modality. The association between the type of treatment and mortality was then assessed after adjustment for recipient age, sex, duration of diabetes, number of antihypertensive drugs, aspirin and statin use, cardiovascular comorbidity, smoking habits, duration of dialysis, donor age and transplant era (early and recent). Several levels of multivariate adjustment were used for all-cause and CVD- and CAD-related mortality analyses: a univariate analysis including transplant type alone; a partly adjusted analysis (model 1) including transplant type and recipient and transplant factors; and finally a fully adjusted analysis (model 2) including transplant type and recipient, transplant and donor factors. The proportional hazards assumption was tested by graphical checks [23]. Smoking was the only variable with missing data $(9 \%)$; the main survival analyses were performed after replacing missing data for smoking using multiple imputations. Missing values were estimated based on known covariates and outcome variables, and 20 sets were created and pooled for analysis. Cox regression analyses were also repeated after exclusion of data for smoking, which did not change our results. All reported $p$ values were two-tailed; $p$ values $<0.05$ were considered significant. Statistical analyses were conducted using IBM SPSS Statistics for Windows, version 21 (IBM, Armonk, NY, USA) or Stata version 13.0 (StataCorp LP, College Station, TX, USA).

\section{Results}

All-cause and CVD- and CAD-related mortality Characteristics of the study population are presented in Table 1. The median follow-up time after transplantation was 7.9 years (IQR 4.3, 12.9), with a maximum follow-up time of 30.6 years. All-cause and cause-specific deaths were analysed by transplant type (Table 2). There were 228 (SPK, $n=100$; LDK, $n=128)$ deaths from all causes, of which 136 (60\%) (SPK, $n=57$ [42\%]; LDK, $n=79$ [58\%]) were caused by CVD. Among CVDs, CAD was the cause of $86(38 \%)$ deaths (SPK, $n=36$ [42\%]; LDK, $n=50$ [58\%]). KaplanMeier curves for all-cause and CVD- and CAD-related mortality grouped by mode of treatment are presented in Figs 1, 2, and 3. When comparing SPK with LDK recipients, HRs from unadjusted Cox models for all-cause and CVD- and CADrelated mortality were $0.63(95 \%$ CI $0.49,0.82 ; p<0.001)$, 0.58 (95\% CI $0.41,0.82 ; p=0.002)$ and 0.57 (95\% CI 0.37 , $0.88 ; p=0.011$ ), respectively. In multivariate Cox proportional hazards models (Tables 3, 4, and 5), with adjustment for transplant type, recipient age, male sex, duration of diabetes, number of antihypertensive drugs, aspirin and statin use, cardiovascular comorbidity, smoking habits, duration of dialysis and transplant year (model 1), HRs and 95\% CIs for all-cause (Table 3) and CAD-related (Table 5) mortality in SPK recipients were $0.76(0.57,1.03 ; p=0.074)$ and $0.66(0.41,1.06$; $p=0.084)$, respectively, using LDK recipients as reference. CVD-related deaths (Table 4) were significantly decreased: HR 0.64 (95\% CI 0.44, 0.93; $p=0.019)$ for SPK compared with LDK recipients. In model 2 , after additional adjustment for donor age, a significant difference was still seen between SPK and LDK recipients for CVD-related mortality (HR 0.63, 95\% CI 0.40, 0.99; $p=0.047$ ) but not for all-cause (HR 0.81, $95 \%$ CI $0.57,1.16 ; p=0.25$ ) or CAD-related (HR $0.63,95 \%$ CI $0.36,1.12 ; p=0.12)$ mortality. Compared with those who received their grafts between 1983 and 1999, patients transplanted after 2000 had adjusted HRs of $0.51(95 \% \mathrm{CI}$ $0.34,0.77 ; p=0.001), 0.39$ (95\% CI $0.22,0.70 ; p=0.002)$ and $0.37(95 \% \mathrm{CI} 0.17,0.78 ; p=0.009)$ for all-cause and CVD- and CAD-related mortality, respectively.

Pancreas graft function at 1 year and influence on all-cause and CVD- and CAD-related mortality Forty-four SPK recipients had lost their pancreas graft within 12 months post transplant. Those who had a functioning $(n=212)$ or failing $(n=44)$ pancreas graft 1 year post transplant were compared with the LDK recipients $(n=230)$ for mortality outcomes. Kaplan-Meier plots for all-cause mortality by transplant type are shown in Fig. 4. SPK patients with a functioning pancreas graft 1 year post transplant had reduced all-cause (HR 0.65 $[95 \%$ CI 0.44, 0.96]; $p=0.029$ ) and CVD- (HR 0.46 [95\% CI $0.28,0.76] ; p=0.003$ ) and CAD-related (HR $0.46[95 \% \mathrm{CI}$ $0.24,0.86] ; p=0.015)$ mortality compared with the LDK 
Table 1 Patient characteristics

\begin{tabular}{|c|c|c|c|}
\hline \multirow[t]{2}{*}{ Variable } & \multicolumn{2}{|l|}{ Transplant type } & \multirow[t]{2}{*}{$p$ value } \\
\hline & SPK $(n=256)$ & LDK $(n=230)$ & \\
\hline \multicolumn{4}{|l|}{ Recipient factors } \\
\hline Mean age at transplantation, years & $40.6 \pm 7.6$ & $48.4 \pm 13.2$ & $<0.001$ \\
\hline Male sex, $n(\%)$ & $186(73)$ & $151(66)$ & 0.10 \\
\hline Mean duration of diabetes pre-transplant, years & $27.6 \pm 7.9$ & $22.9 \pm 11.4$ & $<0.001$ \\
\hline \multicolumn{4}{|l|}{ Relevant medication pre-transplant } \\
\hline Mean no. of antihypertensive drugs & $2.9 \pm 1.3$ & $2.7 \pm 1.2$ & 0.11 \\
\hline Aspirin, $n(\%)$ & $83(32)$ & $75(33)$ & 0.97 \\
\hline Statin, $n(\%)$ & $110(43)$ & $95(41)$ & 0.71 \\
\hline \multicolumn{4}{|l|}{ Pre-transplant history of } \\
\hline $\mathrm{CAD}, n(\%)$ & $58(23)$ & $66(29)$ & 0.13 \\
\hline CBVD, $n(\%)$ & $22(9)$ & $21(9)$ & 0.84 \\
\hline $\mathrm{PAD}, n(\%)$ & $19(7)$ & $33(14)$ & 0.014 \\
\hline Current or past tobacco use pre-transplant $\mathrm{t}^{\mathrm{a}}, n(\%)$ & $144(61)$ & $122(60)$ & 0.89 \\
\hline Duration of dialysis pre-transplant, years & $0.9(0.5,1.4)$ & $0.6(0.3,1.1)$ & 0.002 \\
\hline Pre-emptive transplantation, $n(\%)$ & $86(34)$ & $85(37)$ & 0.44 \\
\hline Median length of follow-up, years & $8.2(4.3,13.1)$ & $7.9(4.3,12.7)$ & 0.53 \\
\hline \multicolumn{4}{|l|}{ Donor factors } \\
\hline Mean age, years & $31.1 \pm 13.4$ & $49.9 \pm 12.7$ & $<0.001$ \\
\hline \multicolumn{4}{|l|}{ Transplant factors } \\
\hline \multicolumn{4}{|l|}{ HLA-ABDR mismatches, $n(\%)$} \\
\hline $0-2$ & $37(14)$ & $113(49)$ & \\
\hline $3-4$ & $164(64)$ & $92(40)$ & \\
\hline $5-6$ & $55(22)$ & $25(11)$ & $<0.001$ \\
\hline \multicolumn{4}{|l|}{ Maintenance immunosuppressive drugs, $n(\%)$} \\
\hline Prednisolone & $256(100)$ & $229(99)$ & 0.47 \\
\hline \multicolumn{4}{|l|}{ Calcineurin inhibitors } \\
\hline Ciclosporin & $109(43)$ & $180(78)$ & $<0.001$ \\
\hline Tacrolimus & $147(57)$ & $43(19)$ & $<0.001$ \\
\hline \multicolumn{4}{|l|}{ Antimetabolites } \\
\hline Azathioprine & $84(33)$ & $90(39)$ & 0.15 \\
\hline Mycophenolate mofetil & $163(64)$ & $113(49)$ & 0.001 \\
\hline mTOR inhibitors & $0(0)$ & $6(3)$ & 0.011 \\
\hline Kidney graft loss, $n(\%)$ & $62(24)$ & $54(23)$ & 0.85 \\
\hline Pancreas graft loss, $n(\%)$ & $89(35)$ & & \\
\hline \multicolumn{4}{|l|}{ Transplant year, $n(\%)$} \\
\hline 1983-1999 & $108(42)$ & $110(48)$ & \\
\hline $2000-2012$ & $148(58)$ & $120(52)$ & 0.21 \\
\hline
\end{tabular}

Data are presented as mean $\pm \mathrm{SD}$, median (IQR) or frequencies $(\%)$

Pre-emptive transplantation denotes patients receiving a transplant before starting dialysis ${ }^{\text {a S}}$ Smoking data were missing in 19 SPK and 27 LDK recipients

mTOR, mammalian target of rapamycin

group, after adjusting for potential confounders. SPK patients with a functioning pancreas graft did better than SPK patients with a failing pancreas graft for all-cause and CVD-related mortality (HR 0.35 [95\% CI 0.23, 0.56], $p<0.001$; and HR 0.30 [95\% CI $0.17,0.54], p<0.001)$, respectively, but the difference was not statistically significant for CAD-related mortality (HR 0.24 [95\% CI 0.06, 1.04]; $p=0.057$ ). Compared with LDK recipients, all-cause mortality was higher in SPK patients with a failing pancreas graft 1 year post transplant (HR 1.61 [95\% CI 1.01, 2.57]; $p=0.044$ ). The trend 
Table 2 Comparison of causes of death between the SPK and LDK groups

\begin{tabular}{lll}
\hline Cause of death & \multicolumn{2}{l}{ Transplant type } \\
\cline { 2 - 3 } & SPK $(n=256)$ & LDK $(n=230)$ \\
\hline Total deaths & $100(44)$ & $128(56)$ \\
CVD & $57(57)$ & $79(62)$ \\
$\quad$ CAD & $36(36)$ & $50(39)$ \\
Infections & $17(17)$ & $23(18)$ \\
Malignant disease & $7(7)$ & $12(9)$ \\
Other identified cause of death & $19(19)$ & $14(11)$ \\
\hline
\end{tabular}

Data are presented as frequencies $(\%)$

was not statistically different for CVD- (HR 1.42 [95\% CI $0.80,2.52] ; p=0.23$ ) and CAD-related (HR 1.52 [95\% CI $0.75,3.09] ; p=0.25$ ) mortality.

Subgroup analysis An interaction analysis was performed between era and transplant type and between recipient age and transplant type to examine a possible interaction between these variables with respect to the incidence of all-cause and CVD- and CAD-related deaths. For each outcome of interest in this study - all-cause and CVD- and CAD-related deathsall the interaction analyses between era and transplant type were non-significant. The HRs in SPK compared with LDK recipients between recent and early eras were $0.53(95 \% \mathrm{CI}$ $0.28,1.01 ; p=0.052), 0.58$ (95\% CI $0.23,1.47 ; p=0.25)$ and 0.27 (95\% CI $0.07,1.07 ; p=0.063)$, respectively. When analysing a test for interaction between recipient age and transplant type on each outcome, we found no significant interaction for all-cause, CVD-related or CAD-related mortality. The HRs were 0.98 (95\% CI $0.95,1.02 ; p=0.33), 0.96$ $(95 \%$ CI $0.92,1.00 ; p=0.066)$ and $0.96(95 \%$ CI $0.91,1.01$;

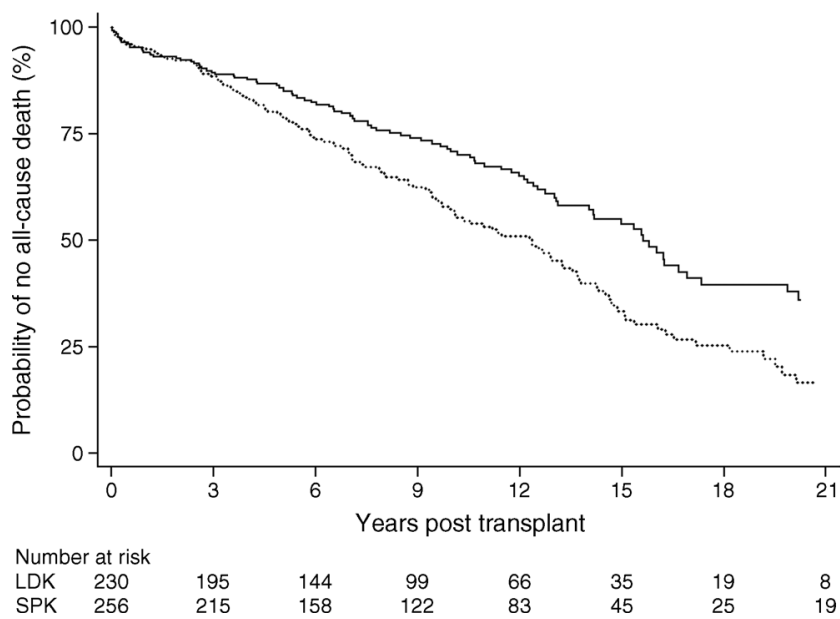

Fig. 1 Kaplan-Meier estimates of probability of no all-cause death, grouped by treatment modality $(p<0.001)$. SPK, solid line; LDK, dotted line

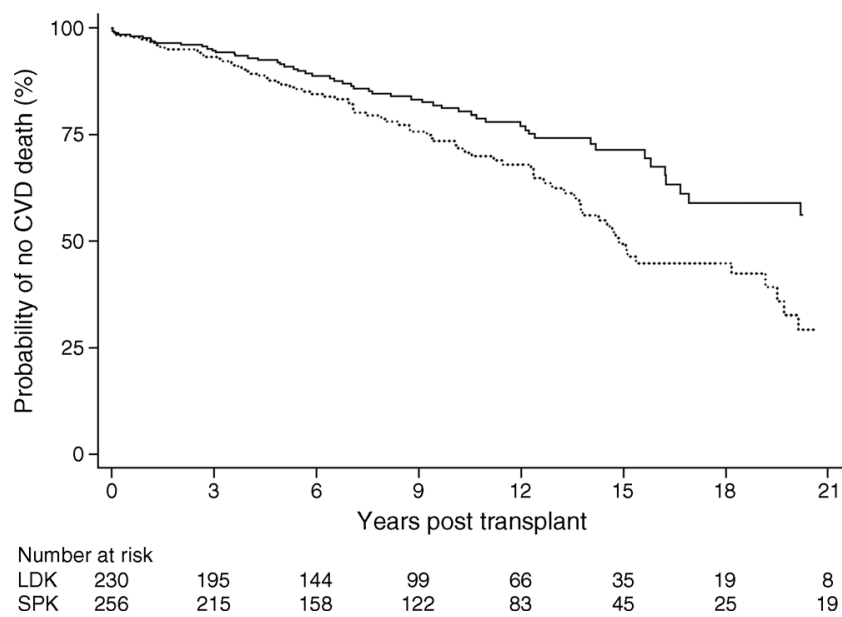

Fig. 2 Kaplan-Meier estimates of probability of no CVD death, grouped by treatment modality $(p=0.002)$. SPK, solid line; LDK, dotted line

$p=0.084)$, respectively, i.e. the effect of age was the same for patients in the SPK and LDK groups. Thus, we did no further subgroup analyses.

\section{Discussion}

In recipients with type 1 diabetes and ESRD we observed that normoglycaemia, as achieved by successful SPK transplantation, was associated with reduced long-term cardiovascular mortality when compared with recipients of an LDK. In addition, reduced cardiovascular mortality was seen in patients who received their grafts from the year 2000 and beyond compared with those transplanted in the early era (19831999).

In general, survival of SPK recipients is higher than survival of recipients of deceased donor KTA [24-30]. However, whether long-term morbidity and survival are better in SPK patients than in LDK patients is still debated. Studies with a

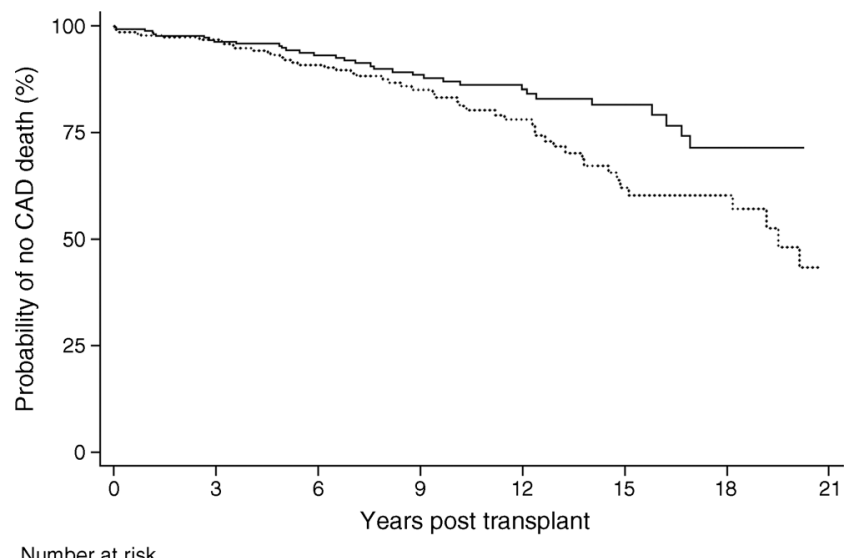

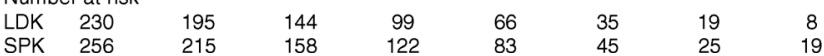

Fig. 3 Kaplan-Meier estimates of probability of no CAD death, grouped by treatment modality $(p=0.011)$. SPK, solid line; LDK, dotted line 
Table 3 HR for death from any cause in SPK vs LDK recipients

\begin{tabular}{|c|c|c|c|c|c|c|c|c|c|}
\hline \multirow[t]{2}{*}{ Variable } & \multicolumn{3}{|c|}{ Unadjusted } & \multicolumn{3}{|c|}{ Adjusted (model 1) } & \multicolumn{3}{|c|}{ Adjusted (model 2) } \\
\hline & HR & $95 \% \mathrm{CI}$ & $p$ value & HR & $95 \% \mathrm{CI}$ & $p$ value & HR & $95 \% \mathrm{CI}$ & $p$ value \\
\hline \multicolumn{10}{|l|}{ Transplant type } \\
\hline LDK & & Reference & & & Reference & & & Reference & \\
\hline SPK & 0.63 & $0.49,0.82$ & $<0.001$ & 0.76 & $0.57,1.03$ & 0.074 & 0.81 & $0.57,1.16$ & 0.25 \\
\hline Recipient age & 1.03 & $1.02,1.05$ & $<0.001$ & 1.04 & $1.02,1.05$ & $<0.001$ & 1.04 & $1.02,1.05$ & $<0.001$ \\
\hline Male sex & 1.18 & $0.89,1.57$ & 0.25 & 1.09 & $0.80,1.47$ & 0.59 & 1.09 & $0.81,1.48$ & 0.57 \\
\hline Diabetes duration & 1.00 & $0.99,1.02$ & 0.70 & 1.00 & $0.99,1.02$ & 0.56 & 1.00 & $0.99,1.02$ & 0.60 \\
\hline No. of antihypertensive drugs & 0.98 & $0.88,1.09$ & 0.66 & 1.09 & $0.96,1.23$ & 0.19 & 1.09 & $0.96,1.23$ & 0.19 \\
\hline Aspirin & 0.99 & $0.74,1.34$ & 0.97 & 0.89 & $0.65,1.23$ & 0.49 & 0.89 & $0.65,1.23$ & 0.49 \\
\hline Statin & 0.63 & $0.46,0.88$ & 0.006 & 0.68 & $0.44,1.04$ & 0.078 & 0.67 & $0.44,1.03$ & 0.071 \\
\hline Cardiovascular comorbidity & 1.60 & $1.21,2.10$ & 0.001 & 1.25 & $0.91,1.73$ & 0.17 & 1.25 & $0.91,1.73$ & 0.18 \\
\hline Smoking history & 1.28 & $0.95,1.70$ & 0.10 & 1.24 & $0.91,1.69$ & 0.17 & 1.24 & $0.91,1.68$ & 0.18 \\
\hline Dialysis duration & 1.03 & $0.86,1.23$ & 0.74 & 1.15 & $0.95,1.40$ & 0.16 & 1.15 & $0.94,1.40$ & 0.16 \\
\hline Donor age & 1.01 & $1.00,1.02$ & 0.003 & & & & 1.00 & $0.99,1.01$ & 0.57 \\
\hline \multicolumn{10}{|l|}{ Transplant year } \\
\hline 1983-1999 & & Reference & & & Reference & & & Reference & \\
\hline $2000-2012$ & 0.53 & $0.39,0.73$ & $<0.001$ & 0.50 & $0.33,0.76$ & 0.001 & 0.51 & $0.34,0.77$ & 0.001 \\
\hline
\end{tabular}

Model 1 is adjusted for transplant type, recipient and transplant factors

Model 2 is additionally adjusted for donor age

Cardiovascular comorbidity includes pre-transplant history of CAD, CBVD and/or PAD

follow-up time of less than 10 years post transplant have shown no difference or a trend towards improved survival with LDK transplantation [10, 12, 29-31]. Studies with follow-up time of more than 10 years after transplantation, however, show trends towards improved outcomes among SPK compared with LDK recipients $[9,11,14,15,28]$.

Kidney transplant recipients have multiple risk factors affecting long-term outcome. In most studies the death-specific causes have not been examined or presented. Since CVD, and particularly ischaemic heart disease, is the leading cause of death in type 1 diabetic patients with ESRD, these specific causes of death may be appropriate endpoints to study in relation to SPK transplantation. There are very few studies that have actually assessed cardiovascular outcomes comparing SPK with KTA. In a small study, Biesenbach et al [32] showed a reduction in risk factors for the development of macroangiopathy but failure to halt progression of macrovascular diseases (CAD, CBVD and PAD) in 11 SPK recipients compared with ten KTA (kidneys from deceased donors) recipients with at least 2 year functioning grafts and a follow-up time of nearly 6 years. However, later on and with a longer follow-up time, the same group showed that the progression of macrovascular diseases was significantly lower in recipients with a functioning SPK graft compared with KTA recipients [33]. Jukema et al [34] compared progression of atherosclerosis (measured by mean-segment diameter loss on coronary angiography) in 26 patients with and six patients without a functioning pancreas after SPK transplantation. Mean follow-up time was 3.9 years. They concluded that progression of atherosclerosis in patients with successful SPK transplantation was reduced compared with recipients with pancreas graft failure. If the progression of macrovascular disease in these studies later translates into cardiac events and increased mortality it would be consistent with our findings. The only study addressing cardiovascular mortality is one that showed a significant reduction in cardiovascular mortality when 130 SPK recipients were compared with 25 patients (originally enrolled on a waiting list for SPK transplantation) who received a KTA and were followed for 7 years [24]. Contrary to our study, their comparator group consisted of recipients receiving a single kidney graft from a deceased donor, which in general is associated with worse outcomes than with an LDK [35]. Thus, our study extends this observation to a benefit of SPK transplantation also when compared with LDK transplantation.

During recent decades there has been substantial improvement in work-up procedures, surgical techniques, immunosuppressive therapy, surveillance and treatment of recipients receiving a kidney transplant. In particular, cardiac assessment and primary as well as secondary prevention of CAD might have improved outcomes. For SPK transplantation, the surgical technique has improved substantially with time, but surgical complications still cause a considerable rate of pancreas graft loss during the first weeks post transplant. Early losses of 
Table 4 HR for cardiovascular death in SPK vs LDK recipients

\begin{tabular}{|c|c|c|c|c|c|c|c|c|c|}
\hline \multirow[t]{2}{*}{ Variable } & \multicolumn{3}{|c|}{ Unadjusted } & \multicolumn{3}{|c|}{ Adjusted (model 1) } & \multicolumn{3}{|c|}{ Adjusted (model 2) } \\
\hline & HR & $95 \% \mathrm{CI}$ & $p$ value & HR & $95 \% \mathrm{CI}$ & $p$ value & HR & $95 \% \mathrm{CI}$ & $p$ value \\
\hline \multicolumn{10}{|l|}{ Transplant type } \\
\hline LDK & & Reference & & & Reference & & & Reference & \\
\hline SPK & 0.58 & $0.41,0.82$ & 0.002 & 0.64 & $0.44,0.93$ & 0.019 & 0.63 & $0.40,0.99$ & 0.047 \\
\hline Recipient age & 1.02 & $1.00,1.03$ & 0.038 & 1.02 & $1.00,1.04$ & 0.027 & 1.02 & $1.00,1.04$ & 0.027 \\
\hline Male sex & 1.36 & $0.93,2.00$ & 0.11 & 1.37 & $0.92,2.04$ & 0.12 & 1.37 & $0.92,2.04$ & 0.12 \\
\hline Diabetes duration & 1.00 & $0.98,1.02$ & 0.64 & 1.00 & $0.98,1.02$ & 0.87 & 1.00 & $0.98,1.02$ & 0.86 \\
\hline No. of antihypertensive drugs & 0.98 & $0.85,1.13$ & 0.80 & 1.09 & $0.93,1.28$ & 0.29 & 1.09 & $0.93,1.28$ & 0.29 \\
\hline Aspirin & 0.93 & $0.63,1.38$ & 0.73 & 1.00 & $0.65,1.52$ & 0.98 & 1.00 & $0.65,1.52$ & 0.99 \\
\hline Statin & 0.42 & $0.26,0.68$ & $<0.001$ & 0.58 & $0.32,1.06$ & 0.079 & 0.58 & $0.32,1.07$ & 0.082 \\
\hline Cardiovascular comorbidity & 1.19 & $0.83,1.73$ & 0.35 & 1.13 & $0.73,1.74$ & 0.59 & 1.13 & $0.73,1.74$ & 0.59 \\
\hline Smoking history & 1.12 & $0.77,1.62$ & 0.55 & 1.04 & $0.70,1.53$ & 0.86 & 1.04 & $0.70,1.53$ & 0.86 \\
\hline Dialysis duration & 0.86 & $0.66,1.11$ & 0.24 & 1.03 & $0.75,1.40$ & 0.86 & 1.03 & $0.80,1.33$ & 0.86 \\
\hline Donor age & 1.01 & $1.00,1.02$ & 0.040 & & & & 1.00 & $0.99,1.01$ & 0.90 \\
\hline \multicolumn{10}{|l|}{ Transplant year } \\
\hline 1983-1999 & & Reference & & & Reference & & & Reference & \\
\hline $2000-2012$ & 0.34 & $0.22,0.54$ & $<0.001$ & 0.39 & $0.22,0.70$ & 0.002 & 0.39 & $0.22,0.70$ & 0.002 \\
\hline
\end{tabular}

Model 1 is adjusted for transplant type, recipient and transplant factors

Model 2 is additionally adjusted for donor age

Cardiovascular comorbidity includes pre-transplant history of CAD, CBVD and/or PAD

Table 5 HR for death from CAD in SPK vs LDK recipients

\begin{tabular}{|c|c|c|c|c|c|c|c|c|c|}
\hline \multirow[t]{2}{*}{ Variable } & \multicolumn{3}{|c|}{ Unadjusted } & \multicolumn{3}{|c|}{ Adjusted (model 1) } & \multicolumn{3}{|c|}{ Adjusted (model 2) } \\
\hline & HR & $95 \% \mathrm{CI}$ & $p$ value & HR & $95 \% \mathrm{CI}$ & $p$ value & HR & $95 \% \mathrm{CI}$ & $p$ value \\
\hline \multicolumn{10}{|l|}{ Transplant type } \\
\hline LDK & & Reference & & & Reference & & & Reference & \\
\hline SPK & 0.57 & $0.37,0.88$ & 0.011 & 0.66 & $0.41,1.06$ & 0.084 & 0.63 & $0.36,1.12$ & 0.12 \\
\hline Recipient age & 1.02 & $1.00,1.04$ & 0.071 & 1.03 & $1.00,1.05$ & 0.043 & 1.03 & $1.00,1.05$ & 0.044 \\
\hline Male sex & 1.52 & $0.93,2.49$ & 0.095 & 1.45 & $0.87,2.44$ & 0.16 & 1.45 & $0.86,2.43$ & 0.17 \\
\hline Diabetes duration & 1.00 & $0.98,1.03$ & 0.72 & 1.01 & $0.99,1.04$ & 0.46 & 1.01 & $0.99,1.04$ & 0.45 \\
\hline No. of antihypertensive drugs & 1.01 & $0.85,1.21$ & 0.88 & 1.11 & $0.91,1.37$ & 0.30 & 1.11 & $0.91,1.37$ & 0.30 \\
\hline Aspirin & 0.81 & $0.48,1.35$ & 0.41 & 0.90 & $0.52,1.55$ & 0.70 & 0.90 & $0.52,1.56$ & 0.71 \\
\hline Statin & 0.47 & $0.26,0.84$ & 0.011 & 0.69 & $0.33,1.47$ & 0.34 & 0.70 & $0.33,1.50$ & 0.36 \\
\hline Cardiovascular comorbidity & 1.14 & $0.71,1.82$ & 0.59 & 1.04 & $0.60,1.79$ & 0.90 & 1.04 & $0.60,1.80$ & 0.90 \\
\hline Smoking history & 1.36 & $0.81,2.28$ & 0.24 & 1.24 & $0.73,2.13$ & 0.43 & 1.25 & $0.73,2.14$ & 0.42 \\
\hline Dialysis duration & 0.66 & $0.44,0.97$ & 0.036 & 0.80 & $0.52,1.23$ & 0.31 & 0.80 & $0.52,1.23$ & 0.31 \\
\hline Donor age & 1.01 & $1.00,1.02$ & 0.099 & & & & 1.00 & $0.98,1.02$ & 0.80 \\
\hline \multicolumn{10}{|l|}{ Transplant year } \\
\hline 1983-1999 & & Reference & & & Reference & & & Reference & \\
\hline 2000-2012 & 0.33 & $0.19,0.60$ & $<0.001$ & 0.37 & $0.17,0.78$ & 0.009 & 0.37 & $0.17,0.78$ & 0.009 \\
\hline
\end{tabular}

Model 1 is adjusted for transplant type, recipient and transplant factors

Model 2 is additionally adjusted for donor age

Cardiovascular comorbidity includes pre-transplant history of CAD, CBVD and/or PAD 


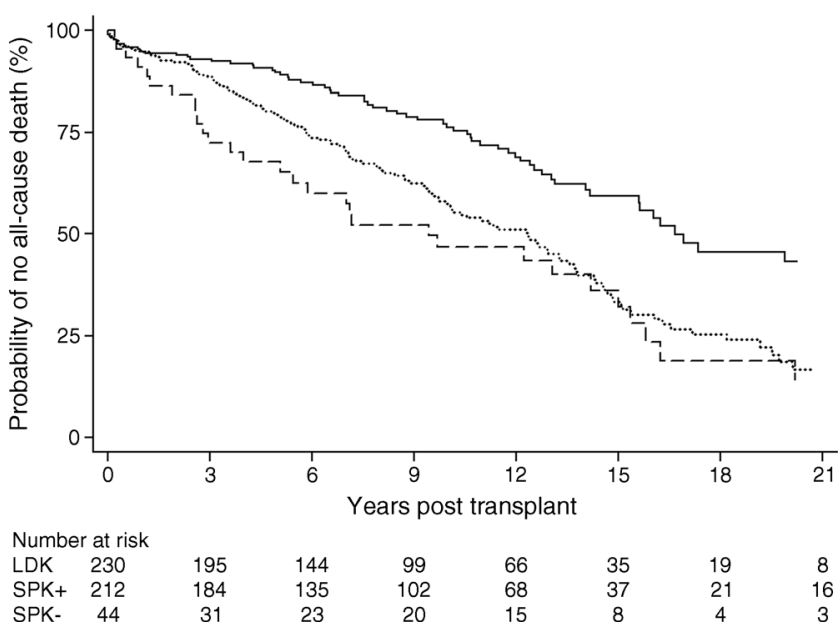

Fig. 4 Kaplan-Meier estimates of probability of no all-cause death by transplant type where the SPK recipients were divided into those with a functioning $(\mathrm{SPK}+)$ or a failing $\left(\mathrm{SPK}^{-}\right)$pancreas graft at 1 year post transplant. SPK+, solid line; SPK-, dashed line; LDK, dotted line. $\mathrm{SPK}+$ compared with LDK recipients $(p<0.001)$; SPK + compared with SPK - recipients $(p<0.001)$; SPK - compared with LDK recipients $(p=0.22)$

the pancreas graft are inversely associated with patient survival. For KTA, graft loss due to early surgical complications is a very rare event. However, in KTA transplantation, higher recipient and living donor age have been accepted in recent years and might have had a negative effect on survival. Obviously it is difficult to adjust for all these changes over time by analysing smaller sub-cohorts, due to loss of statistical power to reveal differences between groups. We therefore analysed the so-called 'vintage effect' by comparing the recipients in an early (1983-1999) and a recent era (2000 2012). Recipients who received their grafts in the early era between 1983 and 1999 had higher all-cause, CVD- and CAD-related mortality compared with the recent era 20002012. This is consistent with improvements in care, as mentioned above. It may also reflect an improvement in cardiovascular work-up, including mandatory coronary angiography for patients with type 1 diabetes prior to transplantation from 1999 and onwards.

The SPK transplant procedure is associated with a higher risk of surgical complications leading to graft loss when compared with LDK transplantation. A pancreas graft loss is inversely associated with long-term survival. As shown by Weiss et al [13], Morath et al [14] and Salvalaggio et al [36], success of dual transplantation (SPK) depends on a long-term functioning pancreas graft. This is consistent with our findings of lower mortality in SPK recipients with a functioning pancreas graft 1 year post transplant compared with those recipients with a failing pancreas graft 1 year post transplant.

The differences in recipient and donor ages between the two groups may introduce a bias. According to our transplantation protocol, recipients offered an SPK transplantation must be less than 55 years of age, but there is no formal upper age limit for KTA. In addition, younger deceased donors are used for SPK grafts. An age bias is therefore unavoidable both for donors and recipients. To try to overcome this, one could analyse outcome of a subgroup of SPK and LDK recipients by age at the time of transplantation after a test for interaction between age and transplant type. However, the relevance of such a subgroup analysis is hampered by low statistical power. The interaction analysis in our study was non-significant. Thus, no further subgroup analysis was performed. In any case, adjusting for age in the Cox models is an appropriate analysis to address this question.

CVD is common after kidney transplantation and is much higher than in the general population [37]. Immunosuppressive agents (i.e. prednisolone, ciclosporin and tacrolimus) have several detrimental effects on cardiovascular risk, including increased risk of hypertension, hyperlipidaemia and post-transplant diabetes mellitus. At our centre, target levels for calcineurin inhibitors have historically been higher in SPK than in LDK recipients. The cardiovascular effects imposed by this higher immunosuppressive load might have moderated the effect of normoglycaemia in SPK recipients.

Limitations of the present study include its retrospective non-randomised design. Also, the extrapolation of our data to non-white individuals may be questioned. We acknowledge that the CVD benefit in SPK recipients is of borderline significance and might have occurred by chance. The strength of our study is the inclusion of a relatively large number of recipients, experience from a single centre, long follow-up time and the fact that no patient was lost to follow-up.

In conclusion, normoglycaemia, compared with moderate hyperglycaemia, was associated with reduced CVD-related mortality in SPK recipients compared with LDK recipients.

Acknowledgements We would like to thank the staff and patients at our institution for their participation.

Funding No specific funding was used for this study.

Duality of interest The authors declare that there is no duality of interest associated with this manuscript.

Contribution statement All authors contributed to each of the following: (1) substantial contribution to the conception and design, acquisition of data and/or analysis and interpretation of data; (2) drafting the article and/or revising it critically for important intellectual content; and (3) final approval of the version to be published. JPL, AH and TJ are guarantors of this work and, as such, had full access to all the data in the study and take responsibility for the integrity of the data and the accuracy of the data analysis. 


\section{References}

1. Foley RN, Parfrey PS, Sarnak MJ (1998) Clinical epidemiology of cardiovascular disease in chronic renal disease. Am J Kidney Dis 32(Suppl 3):S112-S119

2. de Jager DJ, Grootendorst DC, Jager KJ et al (2009) Cardiovascular and noncardiovascular mortality among patients starting dialysis. JAMA 302:1782-1789

3. Collins AJ, Foley RN, Chavers B et al (2012) United States Renal Data System 2011 annual data report: atlas of chronic kidney disease and end-stage renal disease in the United States. Am J Kidney Dis 59(Suppl 1):A7, e1-420

4. Wolfe RA, Ashby VB, Milford EL et al (1999) Comparison of mortality in all patients on dialysis, patients on dialysis awaiting transplantation, and recipients of a first cadaveric transplant. N Engl J Med 341:1725-1730

5. Lentine KL, Brennan DC, Schnitzler MA (2005) Incidence and predictors of myocardial infarction after kidney transplantation. $\mathrm{J}$ Am Soc Nephrol 16:496-506

6. Ojo AO, Hanson JA, Wolfe RA, Leichtman AB, Agodoa LY, Port FK (2000) Long-term survival in renal transplant recipients with graft function. Kidney Int 57:307-313

7. Aakhus S, Dahl K, Wideroe TE (2004) Cardiovascular disease in stable renal transplant patients in Norway: morbidity and mortality during a 5-yr follow-up. Clin Transpl 18:596-604

8. Gruessner AC (2011) 2011 update on pancreas transplantation: comprehensive trend analysis of 25,000 cases followed up over the course of twenty-four years at the International Pancreas Transplant Registry (IPTR). Rev Diabet Stud 8:6-16

9. Sollinger HW, Odorico JS, Becker YT, D'Alessandro AM, Pirsch JD (2009) One thousand simultaneous pancreas-kidney transplants at a single center with 22-year follow-up. Ann Surg 250:618-630

10. Sutherland DE, Gruessner RW, Dunn DL et al (2001) Lessons learned from more than 1,000 pancreas transplants at a single institution. Ann Surg 233:463-501

11. Morath C, Zeier M, Dohler B, Schmidt J, Nawroth PP, Opelz G (2008) Metabolic control improves long-term renal allograft and patient survival in type 1 diabetes. J Am Soc Nephrol 19:1557-1563

12. Young BY, Gill J, Huang E et al (2009) Living donor kidney versus simultaneous pancreas-kidney transplant in type I diabetics: an analysis of the OPTN/UNOS database. Clin J Am Soc Nephrol 4:845-852

13. Weiss AS, Smits G, Wiseman AC (2009) Twelve-month pancreas graft function significantly influences survival following simultaneous pancreas-kidney transplantation. Clin J Am Soc Nephrol 4:988-995

14. Morath C, Zeier M, Dohler B et al (2010) Transplantation of the type 1 diabetic patient: the long-term benefit of a functioning pancreas allograft. Clin J Am Soc Nephrol 5:549-552

15. Lindahl JP, Hartmann A, Horneland R et al (2013) Improved patient survival with simultaneous pancreas and kidney transplantation in recipients with diabetic end-stage renal disease. Diabetologia 56:1364-1371

16. van Dijk PC, Jager KJ, de Charro F et al (2001) Renal replacement therapy in Europe: the results of a collaborative effort by the ERAEDTA registry and six national or regional registries. Nephrol Dial Transplant 16:1120-1129

17. Witczak BJ, Hartmann A, Jenssen T, Foss A, Endresen K (2006) Routine coronary angiography in diabetic nephropathy patients before transplantation. Am J Transplant 6:2403-2408

18. Scanlon PJ, Faxon DP, Audet AM et al (1999) ACC/AHA guidelines for coronary angiography. A report of the American College of Cardiology/American Heart Association Task Force on practice guidelines (Committee on Coronary Angiography). Developed in collaboration with the Society for Cardiac Angiography and Interventions. J Am Coll Cardiol 33:1756-1824

19. Brekke IB (1991) Indications and results of pancreatic transplantation: the Oslo experience 1983-1990. Diabetologia 34(Suppl 1): S18-S20

20. Brekke IB (1999) Pancreas transplantation - a review. Tidsskr Nor Laegeforen 119:3305-3309 [article in Norwegian]

21. Horneland R, Paulsen V, Lindahl JP et al (2015) Pancreas transplantation with enteroanastomosis to native duodenum poses technical challenges - but offers improved endoscopic access for scheduled biopsies and therapeutic interventions. Am J Transplant $15: 242-250$

22. Oyen O, Scholz T, Hartmann A, Pfeffer P (2006) Minimally invasive kidney transplantation: the first experience. Transplant Proc 38:2798-2802

23. Kleinbaum DG, Klein M (2012) Survival analysis: a self-learning text, 3rd edn. Springer, New York

24. La Rocca E, Fiorina P, di Carlo V et al (2001) Cardiovascular outcomes after kidney-pancreas and kidney-alone transplantation. Kidney Int 60:1964-1971

25. Cohen DJ, St Martin L, Christensen LL, Bloom RD, Sung RS (2006) Kidney and pancreas transplantation in the United States, 1995-2004. Am J Transplant 6:1153-1169

26. Mai ML, Ahsan N, Gonwa T (2006) The long-term management of pancreas transplantation. Transplantation 82:991-1003

27. Smets YF, Westendorp RG, van der Pijl JW et al (1999) Effect of simultaneous pancreas-kidney transplantation on mortality of patients with type-1 diabetes mellitus and end-stage renal failure. Lancet 353:1915-1919

28. Becker BN, Brazy PC, Becker YT et al (2000) Simultaneous pancreas-kidney transplantation reduces excess mortality in type 1 diabetic patients with end-stage renal disease. Kidney Int 57:2129 2135

29. Reddy KS, Stablein D, Taranto S et al (2003) Long-term survival following simultaneous kidney-pancreas transplantation versus kidney transplantation alone in patients with type 1 diabetes mellitus and renal failure. Am J Kidney Dis 41:464-470

30. Rayhill SC, D’Alessandro AM, Odorico JS et al (2000) Simultaneous pancreas-kidney transplantation and living related donor renal transplantation in patients with diabetes: is there a difference in survival? Ann Surg 231:417-423

31. Ojo AO, Meier-Kriesche HU, Hanson JA et al (2001) The impact of simultaneous pancreas-kidney transplantation on long-term patient survival. Transplantation 71:82-90

32. Biesenbach G, Margreiter R, Konigsrainer A et al (2000) Comparison of progression of macrovascular diseases after kidney or pancreas and kidney transplantation in diabetic patients with end-stage renal disease. Diabetologia 43:231-234

33. Biesenbach G, Konigsrainer A, Gross C, Margreiter R (2005) Progression of macrovascular diseases is reduced in type 1 diabetic patients after more than 5 years successful combined pancreaskidney transplantation in comparison to kidney transplantation alone. Transpl Int 18:1054-1060

34. Jukema JW, Smets YF, van der Pijl JW et al (2002) Impact of simultaneous pancreas and kidney transplantation on progression of coronary atherosclerosis in patients with end-stage renal failure due to type 1 diabetes. Diabetes Care 25:906-911

35. Wolfe RA, Roys EC, Merion RM (2010) Trends in organ donation and transplantation in the United States, 1999-2008. Am J Transplant 10:961-972

36. Salvalaggio PR, Dzebisashvili N, Pinsky B et al (2009) Incremental value of the pancreas allograft to the survival of simultaneous pancreas-kidney transplant recipients. Diabetes Care 32:600-602

37. Jardine AG, Gaston RS, Fellstrom BC, Holdaas H (2011) Prevention of cardiovascular disease in adult recipients of kidney transplants. Lancet 378:1419-1427 